\title{
Developments in world aquaculture, feed formulations, and role of carotenoids
}

\author{
Samuel P. Meyers \\ Food Science Department, Louisiana State University, Baton Rouge, LA \\ 70806, USA
}

\begin{abstract}
Aquaculture is a rapidly growing global industry, comprising cultivation of various freshwater and marine species of finfish, shellfish, molluscs, and ornamental fish. By the year 2000 , as much as $20 \%$ of the world production of fish will be based on aquaculture. Properly formulated feeds are a significant part of successful aquaculture. Carotenoids play a major role in culture of salmonids such as Atlantic salmon and rainbow trout. Various biological and nutritional roles for carotenoids in salmonids are documented along with the effect of biotic and abiotic factors on astaxanthin transport and retention and final flesh pigmentation. Relevant recent feeding results are presented.
\end{abstract}

\section{INTERNATIONAL DIMENSIONS OF AQUACULTURE}

\section{Global production and growth}

Aquaculture, involving farming of a variety of freshwater and marine species of finfish, shellfish, molluscs, and ornamental fishes, is a rapidly growing global industry with a broad range of technological and managerial practices. In 1990 (ref. 1), world aquaculture reached 15.3 million metric tons (MT), or 12.1 million MT, if aquatic plants were excluded. This is more than double that reported for the year 1975 . Of this, approximately $50 \%$ was of freshwater origin, largely cyprinid species, while $6 \%$ was of marine origin. As much as $80 \%$ of aquaculture production is centered in Asia, particularly in China and Southeast Asia. During the 1984-89 period, capture fisheries expanded only about $14 \%$, while aquaculture rose nearly $70 \%$. Furthermore, the total value of world aquaculture production averaged an annual increase of $15 \%$ during the $1984-90$ period. Market demand for quality product has stimulated much of the growth in aquaculture, especially for salmonids and marine shrimp species. The total world aquatic harvest in 1991, including aquaculture, was about 96 million MT of which aquaculture comprised about $16 \%$ (ref. 2). Harvest limitations in global fisheries suggest that further growth in aquatic production will depend upon aquaculture. Projections are for global aquaculture output to exceed 20 million MT by the year 2000 , comprising about $25 \%$ of the world's seafood.

Of cultured finfish, $85 \%$ are non-carnivorous species, mostly cyprinids of various Chinese and Indian carp species. Almost half of the carnivorous species are salmonids, a ratio of about 50:50 salmon and trout. In the period 1986 to 1989 , increases of $100-180 \%$ occurred in production of farmed Atlantic and Pacific salmon, while increases of 20-70\% were recorded for common carp, tilapias, channel catfish, rainbow trout, and various species of marine shrimp, the latter comprising nearly $88 \%$ of crustacean aquaculture production. Global shrimp production alone in 1989 was over $530,000 \mathrm{MT}$, varying in volume and particular species with specific country. Successful cultivation of other marine finfish includes that of yellowtail, sea bass, sea bream, flatfish, grouper, and snapper. Numerous other marine and freshwater finfish are in various stages of evaluation for commercial aquaculture purposes.

\section{Salmonid production}

In 1991, the worldwide annual harvest of salmon was approximately $720,000 \mathrm{MT}$, of which 25$30 \%$ were produced in a variety of aquaculture facilities and enclosure designs. This includes such salmonid species as rainbow trout, Atlantic salmon, chinook salmon, and coho, pink, and chum salmon. The most spectacular growth has been with Atlantic salmon, notably in Norway 
and, within the past several years, in Chile. Since 1979, production in Norway has expanded from approximately 4,000 MT to over 150,000 MT in the early part of this decade.

With anadromous salmonid species, such as the Pacific and Atlantic salmon, the fish exhibit two distinct life stages, one spent in fresh water (egg and juvenile) and the other under sea conditions (adult). This process, designated smoltification, comprises physiological, morphological, and behavioral changes in which the composite effect of nutrition and carotenoid status in all likelihood plays an important role. With manipulation of the many hatchery variables affecting smoltification, survival of hatchery-reared fish in the wild is a subject of increasing importance. It has been suggested that salmon fry, or alevin, from broodstock deprived of dietary carotenoids, may have low survival rates, i.e. $<15 \%$, compared with those from broodstock receiving properly supplemented diets.

\section{Advances in aquaculture, feed processing and nutrition}

In addition to actual culture operations, tremendous growth has occurred in numbers of feed manufacturing facilities worldwide as well as organizations involved in supplying feed ingredients or additives, algae for larval culture, vaccines, antibiotics, disease diagnostics, and other products that may be required for successful aquaculture. Also, there is an increasing number of organizations supplying services, equipment, and engineering skills to meet the particular aquaculture efforts. The fastest growing segment of the international feed industry is that of feed production for aquaculture. From 3.6 million MT in 1988, aquaculture feed manufacture is expected to increase to as much as 14 million MT by the year 2000. Feed costs represent as much as $40-60 \%$ of the total operating expenses in an intensive aquaculture endeavor, especially with carnivorous species such as salmonids.

Significant growth in aquaculture is evident in culture system design and engineering, feed processing technology, and nutrition. A variety of improved aeration practices has significantly enhanced production of fish and crustaceans, leading to establishment of feeding concepts correlated with the amount of supplemental aeration supplied. Rapid strides in engineering have facilitated development of intensive re-circulating systems in which physical factors can be effectively monitored. In feed processing, improvement in extrusion techniques allows more effective feed manufacture, notably conditioning processes in which enhanced gelatinization of grain products imparts improved feed digestibility and superior feed conversion ratios. Equally important characteristics of extruded feeds are pellet technical quality and a high oil absorption capacity needed for high energy diets. Information is rapidly accumulating on nutrient requirements, practical feed formulations, and factors underlying more cost-effective feed conversion ratios. Included are specific investigations of lipid metabolism and essential fatty acid requirements, and the role of ingredients such as phospholipids, especially phosphatidylcholine, in first-feeding diets. In salmonids, numerous investigations have involved carotenoid metabolism, and the biological role of astaxanthin and factors affecting its transport and deposition in muscle tissue, as well as its proposed function in reproductive processes.

Among current issues in salmon nutrition are those of increasing demand for high-energy/ low-pollution feeds, a need for more efficient growth and feed conversion, and lessening of the potentially deleterious impact of high density culture operations on the immediate environment. Equally important is the effect of nutrition on fish health and final product quality. Various vegetable proteins, especially soybean meal, are being evaluated for gradual replacement of the high amount $(>50 \%$ ) of fish meal used in many dietary formulations. Fish meal may represent as much as $60 \%$ of total feed cost, especially when high-quality meals are utilized. Efforts are being made to utilize fisheries by-products as well as locally produced feedstuffs.

\section{CAROTENOIDS IN AQUACULTURE}

Recent review papers on carotenoids and salmonids, with special emphasis on astaxanthin and canthaxanthin, have concerned relevant subjects such as use of Antarctic krill as a feed and carotenoid source (ref. 3), carotenoid deposition and metabolism (ref. 4), biological activities of carotenoids (ref. 5), microbial sources of astaxanthin (ref. 6), pigmentation of rainbow trout 
(ref. 7) and factors affecting variations in salmonid pigmentation (ref. 8). Numerous other publications have focused on the role of carotenoids in ornamental fish and crustaceans (refs. 9-15).

\section{Sources of carotenoids}

A variety of carotenoids, both synthetic and naturally occurring products, are available or are being developed for use in aquaculture. Included are synthetically produced astaxanthin (3,3'-dihydroxy- $\beta, \beta$-carotene-4,4'-dione) and canthaxanthin ( $\beta, \beta$-carotene- $4,4^{\prime}$-dione) and natural materials such as krill, Spirulina, crustacean-meals, marigold, Capsicum, and other xanthophyll-containing vegetable meals. Added to this list are commercially available products of the astaxanthin-rich yeast Phaffia rhodozyma (refs. 6, 16). Another microbial source being considered is the microalga Haematococcus pluvialis (ref. 6).

\section{Carotenoids and ornamental fishes}

Carotenoids are the primary source of pigmentation in ornamental or tropical fish, responsible for various species-related yellow, red, and related colors. Normally these are obtained through carotenoid-containing organisms in the aquatic food chain, but commercial feed ingredients such as yellow corn, corn gluten meal, and alfalfa are used as sources of carotenoids such as zeaxanthin and lutein (ref. 10). Other carotenoid-rich ingredients used are marigold meal (lutein), red pepper (Capsicum sp.) extract (capsanthin) and krill or crustacean meals (astaxanthin) (refs. 11, 12). Canthaxanthin has been shown to be an effective pigmenter for the tropical fish Trichogaster leeri (ref. 9), and especially to enhance reproductive processes.

\section{Carotenoids and crustaceans}

Astaxanthin is the predominant carotenoid in decapod crustacea and has been studied extensively with the prawn Penaeus japonicus (ref. 14). Oxidative pathways suggested for metabolism of dietary carotenoids include that of $\beta$-carotene and zeaxanthin to astaxanthin. In addition, prawns can directly deposit astaxanthin in their tissues. A comparison of dietary astaxanthin, canthaxanthin, and $\beta$-carotene showed astaxanthin to be most effective for prawn pigmentation. All three carotenoids led to the deposition of mainly astaxanthin esters. Dietary astaxanthin is stored in the integument, carapace and epidermis, and hepatopancreas of the prawn. Recently, Chien and Jing (ref. 15) observed a higher rate of survival of $P$. japonicus on astaxanthin-supplemented diets compared with those containing $\beta$-carotene or algal meal. It was postulated that astaxanthin in the prawn may serve as an intracellular oxygen reserve, allowing the crustacean to survive under the hypoxic conditions of pond culture systems. Howell and Matthews (ref. 13) studied discoloration, "blue disease", of farmed tiger prawn Penaeus monodon, attributed to a nutritional lack of carotenoids. The main carotenoids present in $P$. monodon exoskeletons were astaxanthin, astaxanthin esters and $\beta$-carotene. The "blue" pond-raised prawns had a total carotenoid concentration of 4.3-7 ppm compared with $26.3 \mathrm{ppm}$ in the exoskeleton of wild specimens. Other studies (ref. 17) of $P$. monodon have demonstrated effective carapace pigmentation with Spirulina-supplemented diets.

\section{Carotenoids and salmonids}

The pink to red color in salmonid flesh is due to carotenoids of dietary origin. In wild salmonids, the predominant carotenoid is astaxanthin, derived from feeding on carotenoidrich crustaceans. The range of carotenoid levels in wild salmonids will vary, reflecting differences in the prey, fish size, stage of maturity, and specific pigment metabolism. Carotenoid levels may range from $26-39 \mathrm{mg} / \mathrm{kg}$ flesh for sockeye salmon to $8-9 \mathrm{mg} / \mathrm{kg}$ flesh for chinook salmon and $>3 \mathrm{mg} / \mathrm{kg}$ flesh in rainbow trout. In farmed salmonids, astaxanthin and canthaxanthin are supplemented to feed to impart the desired flesh coloration (ref. 4). Rainbow trout fed diets containing synthetic astaxanthin achieved flesh concentrations of approximately 6.5 to $9 \mathrm{mg}$ pigment $/ \mathrm{kg}$ (refs. 4,7). With larger fish, flesh levels of $20-25 \mathrm{mg}$ pigment $/ \mathrm{kg}$ may occur. Feeds used to pigment rainbow trout or Pacific salmon generally contain 50-100 mg pigment $/ \mathrm{kg}$ feed. Flesh pigment levels of 4-5 mg pigment $/ \mathrm{kg}$ are considered to be the minimum to impart acceptable flesh coloration (ref. 18).

\section{Start-feeding diets}

Dependable availability of postlarvae, fry, or fingerlings is a critical factor in fish and crustacean culture. Significant progress has been made, due largely to better understanding of 
nutrition and enrichment diets and the importance of n-3 highly unsaturated fatty acids (HUFA), particularly eicosopentaenoic acid (22:5) and docosahexaenoic acid (22:6). Torrissen (ref. 19) examined the effect of carotenoids in Atlantic salmon eggs and start-feeding diets on survival and growth. Diets supplemented with synthetic astaxanthin and canthaxanthin promoted growth rates during the early start-feeding period. Astaxanthin was the predominant carotenoid present in the eggs. Subsequently, positive effects of astaxanthin and canthaxanthin, as supplements ( $30 \mathrm{mg} / \mathrm{kg}$ diet) to commercial start-feeding diets, on growth of Atlantic salmon fry, have been reported (ref. 20). Studies of early food selection by Atlantic salmon (ref. 21) suggested that the suitability of zooplankton as a first-feeding diet may be due to the carotenoid present which has a positive effect on embryonic development and subsequent fish growth. Levels of astaxanthin in eggs from wild salmon have been reported to be in the range of 2.1-10.1 mg/g eggs (ref. 22). Loss of both canthaxanthin and astaxanthin during yolk-sac absorption suggests metabolism of the carotenoids and a possible provitamin A function. Earlier, Schiedt et al. (ref. 23) reported on the biological function of astaxanthin, canthaxanthin, and zeaxanthin as vitamin A precursors in vitamin A-depleted rainbow trout.

\section{Biological activities of carotenoids in aquatic species}

Increasing supportive evidence is appearing regarding the biological role of astaxanthin in fish metabolism other than that of a pigmenting agent. Functions of carotenoids in salmonids and other aquatic species have been speculated by Tacon (ref. 24) and Craik (ref. 25), and recently extensively reviewed by Torrissen (ref. 5). Carotenoids in general enhance both the nonspecific and specific immune system (ref. 26). Proposed functions include protection against UV light, serving as provitamin A, enhancing tolerance to elevated ammonia levels and low oxygen levels, stimulation of growth, maturation rate, fecundity reaction as a fertilization hormone, and improvement of egg quality.

Elsewhere (ref. 27), the function of astaxanthin as a powerful antioxidant in marine animals has been demonstrated, providing a defense mechanism for attacking active oxygen species. Astaxanthin also is a strong inhibitor of lipid peroxidation. Kurashige et al. (ref. 28) reported activity of astaxanthin in protection of biological membranes from oxidative injury by inhibition of mitochondrial lipid peroxidation. It was shown that astaxanthin functions as a potent antioxidant both in vivo and in vitro. Jyonouchi et al. (ref. 29) reported on the immunomodulating effects of $\beta$-carotene and astaxanthin on mouse lymphocytes, independent of provitamin A activity. Terao (ref. 30) noted that astaxanthin and canthaxanthin, which possess oxo groups at the 4 and 4-positions in the $\beta$-ring, are more effective antioxidants than $\beta$-carotene in free radical stabilization.

Investigations of carotenoid metabolism in Tilapia nilotica showed that astaxanthin protects biological membranes from oxidative injury by inhibiting mitochondrial lipid peroxidation (ref. 31). Ando and Hatana (ref. 32), in studies of the relationship between carotenoids and salmon egg yolk proteins, demonstrated that the carotenoids are bound to lipovitellin, an immediate precursor of egg yolk protein in salmonid eggs. Actomyosins of fish muscle combine with astaxanthin or canthaxanthin, with a high affinity for astaxanthin (refs. 33, 34). Actomyosins from salmonids showed a higher affinity for ketocarotenoids than did those of other fishes except for common mackerel.

\section{Factors affecting carotenoid function in fish}

Among the many factors affecting carotenoid function in fish (refs. 4,5) are pigment source, form and concentration, diet composition, especially fat content, fish size, physiological state and stage of sexual maturation, and genetic background. Researchers have focused on carotenoid digestibility or intestinal absorption and metabolism and excretion, all of which affect dietary carotenoid utilization and retention. Astaxanthin absorption also depends on the concentration used and whether it is provided in its free form or as a diester. The effects of abiotic factors, i.e., temperature and salinity, have also been examined in studies involving both immature and mature salmonids, including female specimens.

No and Storebakken (refs. 35, 36) examined the effect of salinity and water temperature on pigmentation of rainbow trout, using diets prepared with astaxanthin or canthaxanthin in both fresh water and saltwater. Salinity of the rearing water did not affect the carotenoid composition. Dietary carotenoids were deposited unmodified in the flesh. Astaxanthin was mainly 
deposited as astaxanthin in the skins while the carotenoids in the skin of trout fed the canthaxanthin-supplemented diet were canthaxanthin and its reductive metabolites. Studies of pigmentation with astaxanthin at temperatures of $5^{\circ} \mathrm{C}$ and $15^{\circ} \mathrm{C}$ failed to demonstrate differences in total carotenoid retention although the skin of rainbow trout accumulated more carotenoid at the lower temperature. A difference of 30-50\% between retention and digestibility of astaxanthin was noted, indicating the need for further diet formulations and mechanisms to increase astaxanthin utilization.

Foss et al. (ref. 37) and Torrissen (ref. 18) demonstrated that astaxanthin is deposited in the flesh of rainbow trout more effectively than canthaxanthin due to preferred absorption in the digestive tract as well as deposition in the flesh. A combination of both pigments in the diet gave a higher total carotenoid deposition in the flesh than did either one alone. Deposition increased with increasing fish weight. Foss et al. (ref. 37) observed that rainbow trout utilize dietary unesterified astaxanthin 1.3-1.5 times more efficiently than canthaxanthin, a result attributed to differences in digestibility, better ability of muscle actomyosin to bind astaxanthin, and a higher metabolic turnover of canthaxanthin. Values reported for retention of dietary carotenoids in trout vary from 3-18\% for astaxanthin and $2-7 \%$ for canthaxanthin. Dissimilar distributional patterns of carotenoids occur at various life stages: fry and fingerlings deposit carotenoids mainly in the skin; post-juvenile fish deposit carotenoids in the flesh; at sexual maturation, carotenoids are mobilized from the flesh and selectively transferred to the skin and gonads.

\section{SALMONID FEEDING TRIALS WITH DIETS SUPPLEMENTED WITH ASTAXANTHIN AND CANTHAXANTHIN}

Studies of dietary carotenoids in salmonids have focused on carotenoid digestibility, transport and clearance, and deposition and retention. Storebakken and No (ref. 7) summarized data on metabolism and quantitative aspects of pigmentation in farmed rainbow trout. Dietary lipids directly affect carotenoid deposition levels. Free astaxanthin is more effectively utilized than canthaxanthin, with the former giving flesh a more reddish hue than canthaxanthin at comparable flesh concentrations. Absorption occurs in the intestine with conversion into vitamin A mainly in the intestinal wall. Carotenoids are transported in the blood by lipoproteins with the liver being a major organ for carotenoid metabolism. With sexual maturity, carotenoids are transferred from the flesh to skin and gonads. The skin of trout fed diets with astaxanthin mainly contains astaxanthin and its esters.

Investigations of absorption and distribution of ${ }^{14} \mathrm{C}$-labeled canthaxanthin in rainbow trout (ref. 38) showed the skin, muscle, liver, and kidney to have the highest proportion of ${ }^{14} \mathrm{C}$ in the tissues examined, other than the gastrointestinal tract. Specific activity was higher in the serum than in other tissues. Biliary excretion was shown to be a major excretory route of products of canthaxanthin metabolism. This may explain the large discrepancy between absorption of canthaxanthin in the gastrointestinal tract, as measured in digestibility studies, and net retention of the carotenoid in salmonid tissues. An average retention of $6.5 \%$ was reported with apparent digestibility between 39 and $49.5 \%$. Torrissen et al. (ref. 4) calculated a 4-5\% retention using typical salmon feed canthaxanthin levels. Apparent digestibility of carotenoids by salmonids shows large variations, from ca $13 \%$ for astaxanthin dipalmitate with sea trout to $97 \%$ for free astaxanthin with Atlantic salmon. Discrepancies between digestibility and retention values of carotenoids in salmonids suggest that considerably higher levels of these pigments are absorbed by salmon than are deposited and retained in the flesh. Overestimation of carotenoid digestibility may also be due to destruction of carotenoids in the feces.

The capacity for carotenoid deposition in the flesh of rainbow trout varies from start feeding to sexual maturation (ref. 20). Carotenoid flesh accumulation can vary from $6 \mathrm{mg} / \mathrm{kg}$ to as much as $20-25 \mathrm{mg} / \mathrm{kg}$ in large trout. When dietary levels of astaxanthin exceed $50 \mathrm{mg} / \mathrm{kg}$, there is a low corresponding increase in flesh pigmentation. The flesh pigmentation of both Atlantic salmon and rainbow trout follows a saturation curve (ref. 20). During active growth, high concentrations of carotenoids are deposited in the skin. During sexual maturation, carotenoids are redistributed from the flesh to skin and eggs (refs. 4, 39). Differences in utilization of 
canthaxanthin may be attributed to such factors as dissimilar digestibility (ref. 40) and rate of metabolic turnover, as well as the binding ability of the muscle actomyosin (ref. 41). Flesh carotenoid concentrations of about $6 \mathrm{mg} / \mathrm{kg}$ are sufficient to give a satisfactory pink pigmentation for marketable fresh trout (ref. 4).

Storebakken et al. (ref. 42), in epimerization studies of astaxanthin in Atlantic salmon, reported no significant preferential utilization of the three optical isomers tested (Fig. 1). Epimerization did not occur in the flesh at the chiral centres at C-3 and C-3' in astaxanthin. It was concluded

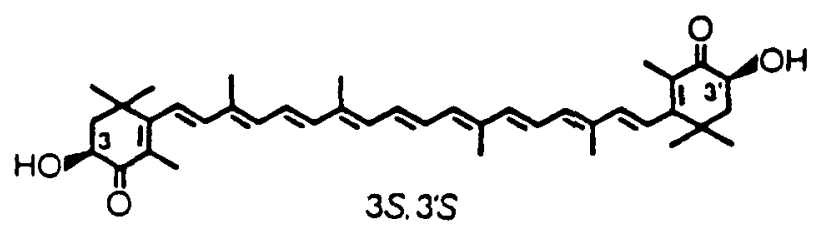

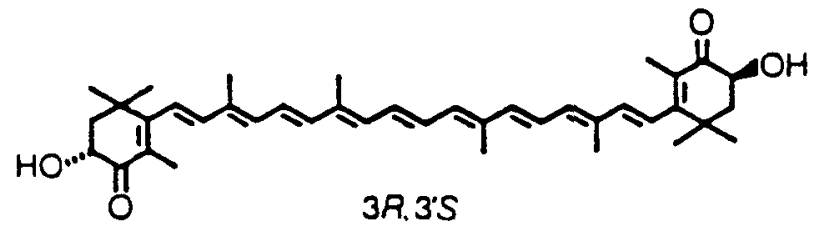<smiles></smiles>

Fig. 1. Optical isomers of astaxanthin.

that the isomeric ratio of astaxanthin in wild salmon reflects the dietary carotenoid deposition rather than a metabolic C-3, 3' epimerization. In studies with rainbow trout, Katsuyama et al. (ref. 43) found dietary astaxanthin diesters mostly absorbed and accumulated in the integument keeping their configurations and partially metabolized to the three stereoisomers of zeaxanthin.

Diets with different optical isomers of astaxanthin [(3S, 3'S), $\left(3 R, 3^{\prime} S\right)$, and $\left.\left(3 R, 3^{\prime} R\right)\right]$ and canthaxanthin have been evaluated with rainbow trout from start feeding to sexual maturation (ref. 20). Astaxanthin stereoisomers deposited in the flesh retained their optical configuration. No significant difference was observed between optical isomer composition of astaxanthin in the flesh and that in feed. Skin of fish fed astaxanthin mainly contained astaxanthin esters while skin of fish fed canthaxanthin contained canthaxanthin and its reductive metabolites. Zeaxanthin in the skin was a mixture of all three optical isomers. The liver and gonads of mature fish contained astaxanthin with an isomeric ratio close to that of the dietary astaxanthin.

Studies using ${ }^{14} \mathrm{C}$-labeled carotenoids with rainbow trout (refs. 44-47) have focused on such aspects as digestibility, transport, accumulation, retention, deposition, and blood clearance. Canthaxanthin ( $130 \mathrm{mg} / \mathrm{kg}$ diet) digestibility was affected by the dietary fat level and antibiotic supplementation (ref. 46). In immature fish, canthaxanthin was present in all lipoprotein fractions in different amounts according to the density of the lipoprotein fraction (ref. 44). When ${ }^{14} \mathrm{C}$-astaxanthin, ${ }^{3} \mathrm{H}$-canthaxanthin, and ${ }^{3} \mathrm{H}$-zeaxanthin were used, astaxanthin levels in blood of mature female trout were higher than those of canthaxanthin, followed by zeaxanthin levels. Blood clearance was similar for all three compounds (ref. 48). Dietary 
canthaxanthin utilization in sexually maturing trout is reported both before and after spawning. Until spawning, canthaxanthin deposition in the flesh was higher in sterile triploids than in diploids. After spawning, carotenoid concentration in diploids reached a level as high as that of triploids after 4 weeks.

\section{CAROTENOID STABILITY AND SENSORY QUALITY OF PROCESSED SALMONID FLESH}

Stability of carotenoids in salmonid flesh under a variety of processing, i.e., cooking, smokecuring, and storage conditions, is an important factor in final product acceptability (refs. 49-51). Color stability of rainbow trout fillets stored under vacuum-packing at $-20^{\circ} \mathrm{C}$ for 6 months has been demonstrated. Significant differences are noted in muscle carotenoid content and degree of hue and lightness in cooked and smoke-cured treatments. Waagbo et al. (ref. 52) have shown relationships between diet and fillet composition and sensory quality of Atlantic salmon with regard to dietary lipids, vitamin $\mathrm{E}$ and the pigment content of the feed. Josephson et al. (ref. 53) examined the flavor profile of cooked salmon and identified several compounds derived from astaxanthin. It was concluded that carotenoids serve as direct precursors to salmon loaf-like flavor or as modulators of chemical reactions which convert fatty acids or other lipid precursors to a salmon aroma. Haard (ref. 54) recently reviewed the effect of physiological and environmental factors on the chemical composition and food quality attributes of cultured fish.

\section{FUTURE DEVELOPMENTS}

All forecasts indicate that global aquaculture will increase significantly by the end of this decade. Advances in diet development and nutrition will include use of alternative proteins in reduction of dietary fish meal and improvement in start-feeding diets for early stages of growth. Significant advances can be anticipated in analysis of nutritional requirements of additional finfish species suitable for commercial culture. Reduction in wild stocks through overfishing or by environmental problems will serve to further stimulate global aquaculture. Along with progress in biotechnology and selection of improved strains of aquaculture species, introduction of (genetically-modified) stock into the natural environment will be an issue of ecological importance. Related will be improvement of particular species in terms of growth, disease resistance, and selection of strains with superior attributes for intensive culture.

Further work in salmonid diet development, especially understanding of lipid metabolism and carotenoid transport, will play a major role in improvement of astaxanthin deposition and retention rates. These investigations will lead to increased understanding of the biological or metabolic role of carotenoids, particularly in young animal start-feeding diets and diets for broodstock of salmonids as well as other finfish species. Availability of alternative sources of astaxanthin, i.e., high-producing strains (>3000 ppm) of the yeast Phaffia rhodozyma, will have a notable impact on developments in salmonid culture. Recent investigations have documented the effective use of this yeast in rainbow trout diets (ref. 55). In view of increased attention being given to the stereoisomer configuration of astaxanthin in wild and cultured salmonids (ref. 20), further studies may prove feasible to determine any possible differences in the physiological functionality of the different isomers of astaxanthin. Finally, aquaculture product quality will be the focus of increased attention, notably nutritional attributes, sensory aspects, and establishment of optimal processing conditions for more effective postharvest utilization. This is particularly applicable with salmonid species where maintenance of optimal flesh pigmentation characteristics is critical to satisfy consumer demand.

\section{REFERENCES}

1. M.B. New, World Aquaculture 22, 28-49 (1991).

2. R.J. Rhodes, Aquaculture Magazine 19, 50-58 (1993).

3. T. Storebakken, Aquaculture 70 193-205 (1988). 
4. O.J. Torrissen, R.W. Hardy, and K.D. Shearer, CRC Crit. Rev. Aquatic Sci. 1, 209-225 (1989).

5. O.J. Torrissen, Proc. Third Int. Symp. on Feeding and Nutr. in Fish pp. 387-399 (1989).

6. E.A. Johnson and G.-H. An, CRC Crit. Rev. Biochem 11, 297-326 (1991).

7. T. Storebakken and H.K. No, Aquaculture 100, 209-229 (1992).

8. G. Choubert, INRA Prod. Anim. 5, 235-246 (1992).

9. M. Fey and S.P. Meyers, I. Aquariculture 1, 15-19 (1980).

10. T. Lovell, Aquaculture Magazine. Sept./Oct. 77-79 (1992).

11. M. Boonyaratpalin and R.T. Lovell, Aquaculture 12, 53-62 (1977).

12. M. Boonyaratpalin and N. Umprasert, Aquaculture 79, 375-380 (1989).

13. B.K. Howell and A.D. Matthews, Comp. Biochem. Physiol. 98B, 375-379 (1991).

14. S. Yamada, Y. Tanaka, M. Sameshima, and Y. Ito, Aquaculture 87 323-330 (1990).

15. Y.-H. Chien and S.-C. Jeng, Aquaculture 102, 333-346 (1992).

16. S.P. Meyers and G.W. Sanderson, Feed Management 43, 12-16, 20 (1992).

17. W.-L. Liao, S.A. Nur-E-Borhan, S. Okada, T. Matsui, and K. Yamaguchi, Nippon Suisan Gakkaishi 59, 165-169 (1993).

18. O.J. Torrissen, Aquaculture 79, 363-374 (1989).

19. O.J. Torrissen, Aquaculture 43, 185-193 (1984).

20. B. Bjerkeng, T. Storebakken, and S. Liaaen-Jensen, Aquaculture 108, 333-346 (1992).

21. J.C. Holm, Aquaculture 54, 173-183 (1986).

22. J.C.A. Craig and S.M. Harvey, I. Fish Biol. 29, 549-565 (1986).

23. K. Schiedt, F.J. Leuenberges, M. Vecchi, and E. Glinz. Pure and Appl. Chem. 57, 685-692 (1985).

24. A.G.J. Tacon, Prog. Fish-Cult. $43,205-208$ (1981).

25. J.C.A. Craig, Aquaculture $47,61-88$ (1985).

26. A. Bendich, L. Nutrition $119,112-115$ (1989).

27. W. Miki, Pure and Appl. Chem. 63, 141-146 (1991).

28. M. Kurashige, E. Okimasu, M. Inoue, and K. Utsumi, Physiol. Chem. Phys. Med. NMR, 22 27-38 (1990).

29. H. Jyonouchi, R.J. Hill, Y. Tomito, and R.A. Good, Nutr. and Cancer, 16, 93-105 (1991).

30. J. Terao, Lipids, 24, 659-661 (1989).

31. M. Katsuyama and T. Matsuno, Comp. Biochem. Physiol. 90B, 131-139 (1988).

32. S. Ando and M. Hatana, Comp. Biochem. Physiol, 99B, 341-344 (1991).

33. H. Henmi, M. Hata, and M. Hata, Nippon Suissan Gakkaishi 56, 1821-1823 (1990).

34. H. Henmi, M. Hata, and M. Takeuchi, Comp. Biochem. Physiol, 99B, 609-612 (1991).

35. H.K. No and T. Storebakken, Aquaculture 101, 123-134 (1992).

36. H.K. No and T. Storebakken, Aquaculture 97, 203-216 (1991).

37. P. Foss, T. Storebakken, E. Austreng, and S. Liaaen-Jensen, Aquaculture 65, 293-305 (1987).

38. R.W. Hardy, O.J. Torrissen, and T.M. Scott, Aquaculture 87, 331-340 (1990).

39. G. Choubert and J.-M. Blanc, Aquaculture 83 359-366 (1989).

40. G. Choubert and T. Storebakken, Aquaculture 81, 69-77 (1989).

41. H. Henmi, T. Iwata, M. Hata, and M. Hata, Tohoku. I. Agric. Res. 37 101-111 (1987).

42. T. Storebakken, P. Foss, E. Austreng, and S. Liaaen-Jensen, Aquaculture 44, 259-269 (1985).

43. M. Katsuyama, T. Komuri, and T. Matsuno, Comp. Biochem. Physiol. 86B 1-5 (1987).

44. G. Choubert, J.-C. G. Milicua, R. Gomez, S. Sance, H. Petit, G.Negre-Sadarques, R. Castillo, and J.-P. Trilles, Comp. Biochem. Physiol. 103A, 403-405 (1992).

45. A. Giullou, G. Choubert, and J. de la Noüe, Comp. Biochem. Physiol. 102B, 61-65 (1992).

46. G. Choubert, J. de la Noüe, and J.-M. Blanc, Aquaculture 29, 323-329 (1991).

47. A. Guillou, G. Choubert, and J. de la Noüe, Comp. Biochem. Physiol. 102B, 61-65 (1992).

48. A. Guillou, G. Choubert, and J. de la Noüe, Comp. Biochem. Physiol. 103A 301-306 (1992).

49. H.K. No and T. Storebakken, I. Food Sci. 56, $969-972$ and 984 (1991).

50. G. Choubert, J.-M. Blanc, and C. Courvalin, Int. I. Food Sci. and Technol. 27, 277-284 (1992).

51. G. Skrede, T. Storebakken, and T. Naes, I. Food Sci. 55, 1574-1578 (1990).

52. R. Waagbo, K. Sandnes, O.J. Torrissen, A. Sandrin, and Ø. Lie, Food Chem. 46, 316-366 (1993).

53. D.P. Josephson, R.C. Lindsay, and D.A. Stuiber, Food Sci. and Tech. 24, 424-432 (1991).

54. N.F. Haard, Food Research Int. 25, 389-307 (1992).

55. F.P. Binkowski, J.J. Sedmak, and S.O. Jolly, Aquaculture Magazine 19, 54-59 (1993). 